\title{
Numerical methods for nonlinear simulations of cyclokinetics illustrating the breakdown of gyrokinetics at high turbulence levels Zhao Deng ${ }^{\text {a, }}$ and R. E. Waltz ${ }^{\text {b }}$ \\ ${ }^{a}$ State Key Laboratory of Nuclear Physics and Technology, School of Physics, Peking University, Beijing 100871, China \\ ${ }^{b}$ General Atomics, P.O. Box 85608, San Diego, California 92186-5608 USA
}

\begin{abstract}
This paper presents the numerical methods for the nonlinear simulations of cyclokinetic [R. E. Waltz and Zhao Deng, Phys. Plasmas 20, 012507 (2013)] equations in Fourier harmonics of the gyro-phase. A parallel processed, implicit time advanced, Eulerian (or continuum) code (rCYCLO) is developed. A novel numerical treatment of the magnetic moment velocity space derivative operator guarantees accurate conservation of the incremental entropy. By comparing the cyclokinetic simulations with the corresponding gyrokinetics, we quantitatively test the breakdown of gyrokinetics at high turbulence levels over a range of large relative ion cyclotron frequency $\left(10<\Omega^{*}<100\right.$ where $\Omega^{*}=1 / \rho^{*}$, and $\rho^{*}$ is the relative ion gyroradius). As an important code verification, the rCYCLO gyrokinetic transport recovers cyclokinetic transport at high relative ion cyclotron frequency $\left(\Omega^{*} \geq 50\right)$ and low turbulence levels. In the case of linearly stable ion cyclotron modes, the cyclokinetic transport is lower (not higher) than the gyrokinetic transport at high turbulence levels and low- $\Omega^{*}$ values.
\end{abstract}

Keywords: Turbulence; Cyclokinetics; Gyrokinetics; Drift-waves; Ion cyclotron modes.

\footnotetext{
* Corresponding author.

E-mail address: zhao.deng@foxmail.com (Zhao Deng).

Cell Phone: (+1)8582301630
} 


\section{Introduction and summary}

In recent years, gyrokinetic simulations of some near edge L-mode tokamak plasmas with the GYRO code ${ }^{1}$ under predict both the transport and the turbulence levels by at least 5 -fold ${ }^{2}$ or even 10 -fold in the more turbulent and colder edge ${ }^{3}$. This missing transport and turbulence (so called "L-mode near edge short fall") suggests that either some important mechanism is missing from current gyrokinetic codes like GYRO, or the gyrokinetic approximation itself is breaking down. It is known that GYRO drift-kinetic ion simulations with gyro-averaging suppressed can recover most of the missing transport ${ }^{3}$. With these motivations, we have developed a 4D $\left[k_{x}, k_{y}, \mu, \alpha\right]$ reduced nonlinear cyclokinetic ${ }^{4}$ code $\mathrm{rCYCLO} . k_{x}, k_{y}$ are the wave-number Fourier transform of the cross-field space positions $x, y, \mu$ is magnetic moment, and $\alpha$ is gyro-phase. The $2 \mathrm{D}\left[k_{z}, v_{z}\right]$ parallel motion is suppressed for simplicity but there are no approximations in the $E \times B$ nonlinearity. Cyclokinetics dynamically follows the high-frequency gyro-motion with no averaging over the gyro-phase $\alpha$. Cyclokinetic simulations nonlinearly couple the high-frequency ion cyclotron modes to the low-frequency drift modes, possibly interrupting and suppressing the gyro-averaging. Nonlinear cyclokinetics has no limit on the amplitude of the perturbations, whereas gyrokinetics is limited to the low drift-wave frequencies and weak turbulence levels (with the perturbed $E \times B$ velocity much less than the ion thermal velocity $\delta v_{\perp}^{E}<<v_{i}^{\text {th }}$ on the small scale of the ion gyroradius $\rho_{i} \cdot k_{\perp} \sim O(1)$ in Ref. [5] p. 113). In summary, gyrokinetics is limited to weak turbulence levels $\delta v_{\perp}^{E}<<v_{i}^{\text {th }}$ and low drift wave frequencies $\omega<<\Omega$, while cyclokinetics extends gyrokinetics to high turbulence levels $\delta v_{\perp}^{E} \leq v_{i}^{\text {th }}$ and high frequencies $\omega \leq N \Omega . \Omega$ is ion cyclotron frequency, and $N$ is the largest ion cyclotron harmonic retained.

rCYCLO code is able to solve both the gyrokinetics and cyclokineitcs independently as initial value problems. Therefore, we can test the breakdown of gyrokineitcs at high turbulence level against cyclokinetics through simulating their turbulent transport at the same geometry, physical parameters, and grid parameters. By comparing 4D $\left[k_{x}, k_{y}, \mu, \alpha\right]$ rCYCLO cyclokinetic transport simulations with the correspondingly reduced 3D $\left[k_{x}, k_{y}, \mu\right]$ gyrokinetic transport simulations, we can quantitatively test the breakdown of gyrokinetics and the gyro-average at high enough turbulence level and low enough relative ion cyclotron frequency $\Omega^{*}$, where $\Omega^{*}=1 / \rho^{*}$ and $\rho^{*}$ is the relative ion gyroradius. We hoped to find the cyclokinetic transport level higher than gyrokinetics so as to qualitatively explain the missing L-mode near edge transport. Instead, we found cyclokinetic transport level lower than gyrokinetics. rCYCLO considers only the 
electrostatic perturbations in a local flux-tube geometry, with a shearless $B$-field decreasing in the radial $x$ direction. This produces a $y$ directed $\operatorname{grad}-B$ drift. The simulation box size is much larger than the eddy size (typically 10's of ion gyro-radii) and much smaller than the variations in the gradient lengths, which are typically close to the machine minor radius size. The ion density and temperature gradients are fixed. The electrons are treated with the collisional drift wave (CDW) fluid model. The rCYCLO code nonlinearly couples the toroidally ( $\operatorname{grad}-B)$ driven ion temperature gradient (ITG) modes and the CDW modes to the stable ion cyclotron (IC) modes. The unstable IC modes are left to future work.

The purpose of rCYCLO code is to investigate the effect of the nonlinear coupling between the high-frequency ion cyclotron motion and low-frequency drift motion. Therefore, it is important that no approximation on the cross-field nonlinear $E \times B$ motion is made. What kind of mode is linearly driven unstable or what artificial damping is chosen is less important, as long as the same model is used in both cyclokinetics and gyrokinetics. The parallel nonlinearity is one order smaller than the cross-field $E \times B$ nonlinearity since $k_{\|} / k_{\perp}=1 / \Omega^{*}$. Gyrokinetic codes typically omit the parallel nonlinearity. The trapped particle modes and the Landau damping in the parallel direction are not relevant to the breakdown of gyrokinetics. Thus, we omit the parallel motion and parallel variations in rCYCLO.

This paper documents the numerical methods used in rCYCLO which is a parallel processed, Eulerian (or continuum) code. The cyclokinetic equations form a system of coupled linear and nonlinear partial differential equations (PDE) [See Eq. (1)]. The perpendicular wave number $\vec{k}_{\perp}$ modes are linearly independent but nonlinearly coupled. The linear and nonlinear terms have a partial derivative in the cyclic gyro-phase variable $\alpha$ and the nonlinear terms have a partial derivative in the magnetic moment variable $\mu$. The latter is a significant challenge for the novel numerical method proposed. Most importantly the nonlinear terms of cyclokinetics do not take the common form $\hat{z} \cdot \vec{k}_{\perp 1} \times \vec{k}_{\perp 2}$ of the nonlinear gyrokinetics [see Eq. (12)] and the more familiar 2D Navier-Stokes equation. An implicit time linear advance step with a lag time nonlinear step is applied. The implicit time linear advance ensures the accuracy of treating the stiff linear dispersion 
matrix which must span the low-frequency drift modes and the higher frequency IC modes. The cyclokinetics of gyro-phase angle form and the cyclokinetics in cyclotron harmonic form $(\mathrm{CKinCH})$ were given in Ref. [4]. Cyclokinetics in the Fourier harmonic form $(\mathrm{CKinFH})$ with discrete Fourier harmonics of the gyro-phase $\alpha$ is introduced in this paper. Only the CKinFH form is used for nonlinear cyclokinetic simulations in rCYCLO in this work. At least 7 to 15 Fourier harmonics are required to accurately recover the linear rates of the gyrokinetic modes even at the highest wave numbers resolving the ion gyroradius scale. The magnetic moment $\mu$-grids, the corresponding weights, and the novel numerical treatment of $\mu$-derivative operator (in particular) are newly designed to conserve the incremental entropy. The $\mu$-derivative operator enters only in the cyclokinetic $E \times B$ nonlinearity. It must perfectly satisfy a $\mu$-integration by parts rule with zero boundary conditions at $\mu=0$ and infinity, which then yields a certain level of inaccuracy in the $\mu$-derivative operation. This inaccuracy will cause the separated convergences of the odd and even $\mu$-grid, and will be suppressed when either the $\mu$-grid is closely spaced or the low-frequency drift modes are decoupled from the high-frequency IC modes. Although the odd and even $\mu$-grid convergence cannot be completely demonstrated for the $\Omega^{*}=10$ standard case at this point, the deviation of the odd and even $\mu$-grid convergence processes caused by this inaccuracy tends to disappear while raising $\Omega^{*}$ from 10 to 30 .

As a linear code validation check of rCYCLO, the CKinFH linear rates from the implicit initial value time advance are in excellent agreement with the eigenvalue solver results for $\mathrm{CKinFH}$ and also for $\mathrm{CKinCH}$. The low-frequency cyclokinetic linear mode rates are in good agreement with the gyrokinetic rates unless $\Omega^{*}$ is smaller than 5. As a nonlinear verification, the rCYCLO gyrokinetic transport level recovers the cyclokinetic transport at high $\Omega^{*}$ value and low turbulence level as required. However for the stable IC modes, the cyclokinetic transport level is lower than the gyrokinetic transport at low- $\Omega^{*}$ value and high turbulence level. The turbulence is drained in frequency space from the unstable low-frequency domain and spreads into the less unstable high-frequency domain. The transport level of cyclokinetics is lower than that of gyrokinetics because the turbulence is drained to the less unstable (and in fact stable) IC modes.

The linear cyclokinetic theory dates to early $60 \mathrm{~s}^{6,7,8}$, although the designation "cyclokinetics" and nonlinear formulation were first introduced in Ref. [4]. Ref. [6] treated the high-frequency current driven ion cyclotron modes, and Refs. [7,8] were among the first to treat low-frequency 
drift modes in linear electrostatic 6D kinetics which integrated over the gyro-phase. Our work is the first to present the numerical simulation of nonlinear cyclokinetic theory for ions. It is also the first to completely solve the ion gyro-phase motion in a nonlinear turbulence system. The numerical method proposed in this work should be useful to a more comprehensive and physically realistic 6D Eulerian kinetic simulation code.

In the present work, our main purpose is to document the numerical methods for cyclokinetic nonlinear simulations and to verify the code rCYCLO. We make a statement of the basic definitions of variables, formulations, coordinate and geometry in Section 2. The numerical methods used in rCYCLO, including the $\mu$-grids discretization, corresponding weights, a novel numerical treatment of $\mu$-derivative operator, the linear eigenvalue solver, and the implicit time advance method are described in Section 3. Simulation results with grid and time-step convergence tests, code verifications and physics discussion are presented in Section 4. Conclusions about numerical methods are contained in Section 5.

\section{Formulations}

\subsection{Definitions}

We follow the notation and analysis of Ref. [4]. Table 1 summarizes the basic units used in rCYCLO. Variables are normalized to gyroBohm units. Any macro-length is scaled to $a$, cross-field micro-turbulence length to sound speed ion gyro-radius $\rho_{s}$, general velocities to ion sound speed $c_{s}$, time to $a / c_{s}$, and ion particle velocity to ion thermal speed $v_{i}^{\text {th }}=\sqrt{2 T_{i} / m_{i}} \cdot B$ is magnetic field. In Cartesian coordinates the cross-field wave-number vector is $\vec{k}_{\perp}=k_{\perp}\left[\cos (\beta) \hat{\varepsilon}_{x}+\sin (\beta) \hat{\varepsilon}_{y}\right]$ with $\hat{\varepsilon}_{y}=\hat{\varepsilon}_{z} \times \hat{\varepsilon}_{x}, \quad \hat{\varepsilon}_{z}=\hat{b}=\vec{B} / B$, and $\beta$ is the wave angle. The three-wave interaction requires $\vec{k}_{2}=\vec{k}-\vec{k}_{1}$. The perpendicular ion velocity can be expressed as $\vec{v}_{\perp}=v_{\perp}\left(\hat{\varepsilon}_{x} \cos \alpha+\hat{\varepsilon}_{y} \sin \alpha\right)$ where $\alpha$ is the gyro-phase angle with $0 \leq \alpha<2 \pi$. The ion magnetic moment is $\mu=m_{i} v_{\perp}^{2} / 2 B$. We normalize the wave number, time, and magnetic moment as 
$\hat{k}=k_{\perp} \rho_{s}, \hat{t}=t\left(c_{s} / a\right)$, and $\hat{\mu}=\left(v_{\perp} / v_{i}^{t h}\right)^{2}$ respectively. The perturbed ion distribution function, electric potential, and two-dimensional Maxwell ion distribution function are normalized as $\delta \hat{f}_{k}=\left(\delta f_{k} T_{i} / m_{i}\right) / \rho^{*}, \quad \delta \hat{\phi}_{k}=\left(e \delta \phi_{k} / T_{e}\right) / \rho^{*}, \quad$ and $\quad F_{M}(\hat{\mu})=e^{-\hat{\mu}} / 2 \pi \quad$ respectively, where $\rho^{*}=\rho_{s} / a$ is the relative ion gyro radius. The velocity space integration of the two-dimensional Maxwell ion distribution function is $\oint d \alpha \int_{0}^{\infty} d \hat{\mu} F_{M}(\hat{\mu})=1$ where the parallel velocity has been suppressed. The normalized perturbed ion density is given by $\delta \hat{n}_{k}^{i}=\oint d \alpha \int_{0}^{\infty} d \hat{\mu} \delta \hat{f}_{k}(\hat{\mu}, \alpha) / n_{0}$ where $n_{0}$ is the unperturbed ion density. Notice that the distribution function is cyclic in the gyro-phase angle: $\delta \hat{f}_{k}(\hat{\mu}, \alpha+2 \pi)=\delta \hat{f}_{k}(\hat{\mu}, \alpha)$ on $0 \leq \alpha<2 \pi$.

Table 1

Summary of basic units in rCYCLO

\begin{tabular}{lcl}
\hline Dimension & Unit & Description \\
\hline Macro length & $a$ & Tokamak minor radius \\
Micro length & $\rho_{s}=c_{s} / \Omega$ & Sound speed ion gyro-radius \\
Velocity & $c_{s}=\sqrt{T_{e} / m_{i}}$ & Ion sound speed \\
Frequency & $\Omega=e B / m_{i} c$ & Ion gyro frequency \\
Mass & $m_{i}$ & Ion mass \\
Time & $a / c_{s}$ & \\
Temperature & $T_{e}$ & Electron temperature \\
Density & $n_{e}$ & Electron density \\
Particle flux & $n_{e} c_{s}\left(\rho_{s} / a\right)^{2}$ & \\
Energy flux & $T_{e} n_{e} c_{s}\left(\rho_{s} / a\right)^{2}$ & \\
Diffusivity & $\left(c_{s} / a\right) \rho_{s}^{2}$ &
\end{tabular}




\subsection{Coordinates and geometry}

rCYCLO assumes a two-dimensional local flux-tube geometry $(x, y)$ perpendicular to magnetic field $\vec{B}$. Parallel variations and parallel velocity are suppressed $\partial / \partial_{z} \Rightarrow 0, u_{z} \Rightarrow 0$. The simulation box $(x, y)$ is a small symmetric rectangle of side $L$ with $\rho_{s}<<L<<a$. The straight and shearless magnetic field varies only in the $x$ direction $B(x)=B[1-(x+r) / R] . x$ represents the radial position with $x=0$ at center of the box, $R$ is the major radius of tokamak, and $r$ is the minor radius of the flux-tube. Since no trapped particles are considered, the value of the local inverse aspect ratio $r / R$ does not enter in this work. As a continuum code, rCYCLO transforms real space $(x, y)$ to Fourier wave-number space $\left(k_{x}, k_{y}\right)$. Since the transformed variables satisfy the reality condition $\delta \hat{\phi}_{-k}=\delta \hat{\phi}_{k}^{*}$, and $\delta \hat{f}_{-k}=\delta \hat{f}_{k}^{*}$, rCYCLO only needs to time advance the perturbations in the upper half $k$-plane $0 \leq k_{y} \leq k_{y}^{\max },-k_{x}^{\max } \leq k_{x} \leq-k_{x}^{\max }$, and obtains the lower half $k$-plane $-k_{y}^{\max } \leq k_{y} \leq 0,-k_{x}^{\max } \leq k_{x} \leq-k_{x}^{\max }$ by the conjugate projection.

\subsection{Cyclokinetic equation in gyro-phase angle form}

Following from Eq. (9b) of Ref. [4], the non-conservative form of the collisionless cyclokinetic equation in gyro phase angle space is given by

$$
\begin{gathered}
D \delta \hat{f}_{k} / D \hat{t}-i \hat{\omega}_{k}^{d} \delta \hat{g}_{k}+i \overrightarrow{\hat{v}}_{\perp} \cdot \overrightarrow{\hat{k}} \Omega_{*} \delta \hat{g}_{k}-\Omega_{*} \partial_{\alpha} \delta \hat{g}_{k}=-i \hat{\omega}_{* k}^{n T} \delta \hat{\phi}_{k} n_{0} F_{M}(\hat{\mu}) \\
+\sum_{k 1}\left[\left(T_{e} / T_{i}\right) \delta \hat{\phi}_{k 1} i \overrightarrow{\hat{k}}_{1} \cdot \overrightarrow{\hat{v}}_{\perp} \partial_{\hat{\mu}} \delta \hat{f}_{k 2}+\delta \hat{\phi}_{k 1} \overrightarrow{\vec{k}_{1}} \cdot \hat{b} \times \overrightarrow{\hat{v}}_{\perp} / \hat{v}_{\perp}^{2} \partial_{\alpha} \delta \hat{f}_{k 2}\right]
\end{gathered}
$$

where $\vec{k}_{2}=\vec{k}-\vec{k}_{1} . \quad \delta \hat{g}_{k}(\hat{\mu}, \alpha)=\delta \hat{f}_{k}(\hat{\mu}, \alpha)+\left(T_{e} / T_{i}\right) \delta \hat{\phi}_{k} n_{0} F_{M}(\hat{\mu})$ represents the non-adiabatic part of distribution function. $\hat{\omega}_{k}^{d}=2 \hat{k}_{y}\left(T_{i} / T_{e}\right) \hat{\mu}(a / R)$ is the grad- $B$ drift frequency. We add the factor 2 here to compensate for the curvature drive from the suppressed parallel direction. The $\Omega^{*}$ terms correspond to the linear gyro-motion on top of the linear grad-B motion $\left(\hat{\omega}_{k}^{d}\right.$ term) and the remaining linear and nonlinear $E x B$ motion of the right hand side of Eq. (1). $\hat{\omega}_{*}^{n T}=\hat{k}_{y}\left[\left(a / L_{n}\right)+\left(a / L_{T i}\right)(\hat{\mu}-1)\right]$ is the density and temperature gradient drive frequency for the 
drift-waves. In rCYCLO, artificial damping at high and low $k$ is built into time derivative operator $D / D \hat{t}=\partial / \partial \hat{t}+\mu_{H K} \hat{k}^{4}+\mu_{L K} / \hat{k}^{2} . \mu_{H K}$ and $\mu_{L K}$ are constants. $\mu_{H K} \hat{k}^{4}$ is the damping of high- $k$ modes which allows turbulence energy to escape to short wave length modes where it is physically damped by collisions. $\mu_{L K} / \hat{k}^{2}$ is the damping of low- $k$ modes. It physically represents the neglected magnetic shear damping which makes the low- $k$ modes slightly stable. This allows the low- $k$ modes to saturate the inverse cascade energy from the higher $k$ driving modes. The large factor $\Omega^{*}=1 / \rho^{*}$ is the relative ion cyclotron frequency, which ranges from 10 to 1000 with 50 to 100 being the typically physical values of interest. We set $n_{0} \equiv 1$ in units of density $n_{e}$, which means $n_{i}=n_{e}$.

The Poisson equation is given as

$$
\delta \hat{\phi}_{k}=\left[\oint d \alpha \int_{0}^{\infty} d \hat{\mu} \delta \hat{f}_{k}(\hat{\mu}, \alpha) / n_{0}-\delta \hat{n}_{k}^{e}\right] /\left(\hat{\lambda}_{D}^{2} \hat{k}^{2}\right)
$$

where $\hat{\lambda}_{D}<<1$ is the Debye length in units of $\rho_{s} . \delta \hat{n}_{k}^{e}=\left(\delta n_{k}^{e} / n_{0}\right) / \rho^{*}$ is the normalized perturbed electron density, and $\delta \hat{n}_{k}^{i}=\oint d \alpha \int_{0}^{\infty} d \hat{\mu} \delta \hat{f}_{k}(\hat{\mu}, \alpha) / n_{0}$ is the normalized perturbed ion density. The time advance of the electron perturbations is given in Section 2.7.

The radial ion particle and energy fluxes in gyroBohm units of $n_{0} c_{s} \rho^{* 2}$ and $T_{e} n_{0} c_{s} \rho^{* 2}$ are

$$
\left[\hat{\Gamma}, \hat{Q}^{\perp}\right]=\operatorname{Re} \oint d \alpha \int_{0}^{\infty} d \hat{\mu} \sum_{k}\left[1,\left(T_{i} / T_{e}\right) \hat{\mu}\right] i \hat{k}_{y} \delta \hat{\phi}_{k}^{*}\left[\delta \hat{g}_{k}(\hat{\mu}, \alpha) / n_{0}\right]
$$

where $R e$ means only the real part is retained. $\delta \hat{g}_{k}$ indicates that transport is proportional to the non-adiabatic perturbed distribution function. The diffusivities are defined as fluxes divided by gradients $[\hat{D}, \hat{\chi}]=\left[\hat{\Gamma}, \hat{Q}^{\perp}\right] /\left[a / L_{n}, a / L_{T i}\right]$.

We do not apply Eq. (1) in the simulations since the stiff $\alpha$-derivative term $\Omega_{*} \partial_{\alpha} \delta \hat{g}_{k}$ is difficult to time advance accurately. It is better to transform $\alpha$ to a harmonic space so as to replace the derivative operator by the harmonic number identity. There are two kinds of harmonic transforms: Fourier harmonics and cyclotron harmonics described in Section 2.4 and Section 2.5 respectively. The nonlinear cyclokinetic simulations are done with the Fourier harmonics in this 
paper, while the cyclotron harmonic form is only used for the linear mode rate validation checks.

\subsection{Cyclokinetic equation in Fourier harmonic form (CKinFH)}

After straightforwardly expanding $\delta \hat{f}_{k}(\hat{\mu}, \alpha)$ in Eq. (1) with finite (or discrete) Fourier harmonics $\delta \hat{f}_{k}(\hat{\mu}, \alpha)=\sum_{n=-N_{\alpha}+1}^{n=N_{\alpha}-1} \delta \hat{F}_{k}^{n}(\hat{\mu}) \exp ($ in $\alpha)$. we obtain the 4D cyclokinetic equation in Fourier harmonic form given by Eq. (4) where $n=-N_{\alpha}+1, \ldots 0 \ldots, N_{\alpha}-1$ is the harmonic number with respect to $\alpha$. The corresponding inverse Fourier transform is

$$
\begin{aligned}
& \delta \hat{F}_{k}^{n}(\hat{\mu})=\frac{1}{2 N_{\alpha}-1} \sum_{m=0}^{m=2 N_{\alpha}-2} \delta \hat{f}_{k}\left(\hat{\mu}, \alpha_{m}\right) \exp \left(-i n \alpha_{m}\right) \text {, where } \alpha_{m}=2 \pi m /\left(2 N_{\alpha}-1\right) \text {. } \\
& D \delta \hat{F}_{k}^{n} / D \hat{t}-i \hat{\omega}_{k}^{d} \delta \hat{G}_{k}^{n}+i\left(\hat{v}_{\perp} \hat{k} / 2\right) \Omega_{*}\left[\exp (-i \beta) \delta \hat{G}_{k}^{n-1}+\exp (+i \beta) \delta \hat{G}_{k}^{n+1}\right]-\Omega_{*} i n \hat{G}_{k}^{n}= \\
& -i \hat{\omega}_{* k}^{n T} \delta \hat{\phi}_{k} n_{0} F_{M}(\hat{\mu}) \delta_{0}^{n}+{ }^{N L} S(\hat{k}, \hat{\mu}, n)
\end{aligned}
$$

The non-adiabatic part of the distribution function is $\delta \hat{G}_{k}^{n}=\delta \hat{F}_{k}^{n}+\left(T_{e} / T_{i}\right) \delta \hat{\phi}_{k} n_{0} F_{M}(\hat{\mu}) \delta_{0}^{n}$ with $\delta_{0}^{n}=[0,1]$. The perturbed distribution functions are linearly coupled and satisfy the reality condition $\delta \hat{F}_{-k}^{-n}=\left[\delta \hat{F}_{k}^{n}\right]^{*} .{ }^{N L} S(\hat{k}, \hat{\mu}, n)$ represents the nonlinear dynamics.

The proper cyclic boundary condition on harmonic number $n$ must be applied in order to conserve the incremental entropy. The incremental entropy of CKinFH is defined by

$$
E=\sum_{k} \oint d \alpha \int_{0}^{\infty} d \hat{\mu}\left(\delta \hat{f}_{k}^{*} \delta \hat{f}_{k}\right)=2 \pi \int_{0}^{\infty} d \hat{\mu} \sum_{k} \sum_{n=-N_{\alpha}+1}^{n=N_{\alpha}-1}\left(\delta \hat{F}_{k}^{n}\right)^{*} \delta \hat{F}_{k}^{n}
$$

With the linear terms in Eq. (1) and Eq. (4) deleted, it is easy to show in the gyro-phase form, and with some pains-taking algebra in the discrete Fourier transform form that the total $E$ is conserved.

Since gyro phase angle space is cyclic $\delta \hat{f}_{k}(\hat{\mu}, \alpha+2 \pi)=\delta \hat{f}_{k}(\hat{\mu}, \alpha)$ on $0 \leq \alpha<2 \pi$, its Fourier form $\delta \hat{F}_{k}^{n}$ is also cyclic. For finite harmonic number $n=-N_{\alpha}+1, \ldots 0 \ldots, N_{\alpha}-1$, cyclic boundary condition is provided by $\delta \hat{F}_{k}^{-N_{\alpha}}=\delta \hat{F}_{k}^{N_{\alpha}-1}, \delta \hat{F}_{k}^{N_{\alpha}}=\delta \hat{F}_{k}^{-N_{\alpha}+1}$ as demonstrated from the 
inverse Fourier transform. The derivative operator also needs a Fourier transform $\partial_{\alpha} \delta \hat{f}_{k}(\hat{\mu}, \alpha) \Rightarrow i \bar{n} \delta \hat{F}_{k}^{n}(\hat{\mu})$, where $i \bar{n}(n)$ is the derivative harmonic identity, which is cyclic $i \bar{n}\left[n \pm\left(2 N_{\alpha}-1\right)\right]=i \bar{n}(n) \quad$ and $\quad i \bar{n}(n) \quad$ is $\quad$ calculated $\quad$ by $\quad i \bar{n}(n)=i\left[n-j\left(2 N_{\alpha}-1\right)\right] \quad$ when $-N_{\alpha}+1+j\left(2 N_{\alpha}-1\right) \leq n \leq N_{\alpha}-1+j\left(2 N_{\alpha}-1\right)$ with an integer $j$. So the cyclic boundary condition of the derivative harmonic identity should be $i \bar{n}\left(N_{\alpha}\right)=i \bar{n}\left(-N_{\alpha}+1\right)$ and $i \bar{n}\left(-N_{\alpha}\right)=i \bar{n}\left(N_{\alpha}-1\right)$.

Eq. (1) is in non-conservative form, and we can obtain its conservative form by applying the crucial identity Eq. (6) to Eq. (1).

$$
\left(T_{e} / T_{i}\right) \partial_{\hat{\mu}} \overrightarrow{\hat{k}}_{1} \cdot \overrightarrow{\hat{v}}_{\perp}+\partial_{\alpha} \overrightarrow{\hat{k}} \cdot \hat{b} \times \overrightarrow{\hat{v}}_{\perp} / \hat{v}_{\perp}^{2}=0
$$

After the discrete Fourier harmonic transformation, we get both the conservative and non-conservative form of the nonlinear dynamics. The conservative form is

$$
\begin{aligned}
{ }_{C o n}^{N L} S(\hat{k}, \hat{\mu}, n) & =\partial_{\hat{\mu}} \sum_{k 1}\left(T_{e} / T_{i}\right) \delta \hat{\phi}_{k 1} i\left(\hat{v}_{\perp} \hat{k}_{1} / 2\right)\left[\exp \left(-i \beta_{1}\right) \delta \hat{F}_{k 2}^{n-1}+\exp \left(+i \beta_{1}\right) \delta \hat{F}_{k 2}^{n+1}\right] \\
& -\sum_{k 1} \delta \hat{\phi}_{k 1} i \hat{k}_{1} /\left(2 \hat{v}_{\perp}\right) n\left[\exp \left(-i \beta_{1}\right) \delta \hat{F}_{k 2}^{n-1}-\exp \left(+i \beta_{1}\right) \delta \hat{F}_{k 2}^{n+1}\right]
\end{aligned},
$$

and the non-Conservative form is

$$
\begin{aligned}
{ }_{N C o n}^{N L} S(\hat{k}, \hat{\mu}, n) & =\sum_{k 1}\left(T_{e} / T_{i}\right) \delta \hat{\phi}_{k 1} i\left(\hat{v}_{\perp} \hat{k}_{1} / 2\right)\left[\exp \left(-i \beta_{1}\right) \partial_{\hat{\mu}} \delta \hat{F}_{k 2}^{n-1}+\exp \left(+i \beta_{1}\right) \partial_{\hat{\mu}} \delta \hat{F}_{k 2}^{n+1}\right] \\
& -\sum_{k 1} \delta \hat{\phi}_{k 1} i \hat{k}_{1} /\left(2 \hat{v}_{\perp}\right)\left[\exp \left(-i \beta_{1}\right)(n-1) \delta \hat{F}_{k 2}^{n-1}-\exp \left(+i \beta_{1}\right)(n+1) \delta \hat{F}_{k 2}^{n+1}\right]
\end{aligned} .
$$

Derive the $\partial_{\hat{\mu}}$ into the bracket in the first term of Eq. (7), then the conservative form Eq. (7) and non-conservative form Eq. (8) are found to be equivalent as required. Notice that the nonlinear coupling coefficient is enhanced by $1 / \hat{v}_{\perp}$ at low velocities.

In rCYCLO code, the nonlinear term is calculated by $\left.{ }_{C \text { Con }}^{N L} S(\hat{k}, \hat{\mu}, n)+_{N C o n}^{N L} S(\hat{k}, \hat{\mu}, n)\right] / 2$ in order to cancel the numerical error from the numerical approximation of the $\mu$-derivative operator in the nonlinear conservation of incremental entropy. Numerical nonlinear incremental entropy conservation is a crucial test of any turbulence simulation code (e.g. see Ref. [9] in the case of the GYRO code). Without accurate conservation there may be no nonlinear saturation. The 
discretization of the derivative operator $\partial_{\hat{\mu}}$ and numerical incremental entropy conservation are discussed in detail in section 3.2. As explained in section 3.4, the lag time step and expensive calculation of nonlinear terms requires collective communication from all the four dimensions $[k x$, $k y, \mu, n]$ while the fast parallel process computation of the implicit time step linear terms requires collective communication from only two dimensions $[\mu, n]$ in solving the Poisson equation. The calculation of nonlinear dynamics in Eq. (7), (8) is only parallelized in the $n$ and $\mu$ dimensions, since the communications in $n$ and $\mu$ dimensions are much simpler than those in $k x$ and $k y$ dimensions.

The Poisson equation for CKinFH is given as

$$
\delta \hat{\phi}_{k}=\left[2 \pi \int d \hat{\mu} \delta \hat{F}_{k}^{0}(\hat{\mu}) / n_{0}-\delta \hat{n}_{k}^{e}\right] /\left(\hat{\lambda}_{D}^{2} \hat{k}^{2}\right)
$$

Eq. (9) indicates that only the zero-th Fourier harmonic $\delta \hat{F}_{k}^{0}$ contributes to the electrostatic potential for CKinFH. Whether the Debye length $\hat{\lambda}_{D}$ is a very small number or even zero is not so important for low- $k$ ITG gyrokinetics (unless we are looking at high- $k$ electromagnetic ETG). However it is very important to cyclokinetics, since Eq. (9) is numerically close to " $0 / 0 "$. Moreover, there is a non-zero polarization term in the denominator of the gyrokinetic Poisson equation, while there is no such polarization term in the denominator of CKinFK Poisson equation Eq. (9). (See appendix for the absence of polarization term with all harmonics retained of an equivalent cyclokinetic form.) If the simulation were in real space $(x, y)$, it would likely be impractical to resolve such small scales while on the ion gyro-scale. We would need to extrapolate the physically small $\hat{\lambda}_{D}^{2}$ from some larger and numerically practical $\hat{\lambda}_{D}^{2}$. However $\hat{\lambda}_{D}^{2}$ should not be too large, since an unphysically large $\hat{\lambda}_{D}^{2}$ will produce spurious high- $k$ ITG modes in both gyrokinetics and cyclokinetics. In the rCYCLO simulations, the linear growth rates and frequencies do not significantly depend on the size of $\hat{\lambda}_{D}^{2}$ if it is small enough e.g. 0.01 .

The particle and energy fluxes are given by 


\subsection{Cyclokinetic equation in cyclotron harmonic form (CKinCH)}

Ref. [4] gives linear and nonlinear cyclokinetic equations in the cyclotron harmonic form. The cyclotron harmonic transform is defined by

$$
\delta \hat{f}_{k}(\hat{\mu}, \alpha) \exp [-i k \rho \sin (\alpha-\beta)]=\sum_{n=-\infty}^{n=+\infty} \delta \hat{F}_{k}^{n}(\hat{\mu}) \exp [i n(\alpha-\beta)]
$$

where $\rho=v_{\perp} / \Omega$ is ion gyro radius. (The Eq. (12) of Ref. [4] used $i k \rho \sin (\alpha-\beta$ ), when it should have been $-i k \rho \sin (\alpha-\beta))$. We repeat the equations of $\mathrm{CKinCH}$ in the Appendix for convenience. Unlike the Fourier harmonics, the cyclotron harmonics are the linearly uncoupled "normal modes" of cyclokinetics. We have applied $\mathrm{CKinCH}$ to linearly verify CKinFH. The $\mathrm{CKinCH}$ nonlinear simulations are considerably more expensive than the CKinFH. Only CKinFH is used for nonlinear cyclokinetic simulations in this work.

\subsection{Gyrokinetic equation}

Ref. [4] shows that the $\mathrm{CKinCH}$ equation entirely reduces to the gyrokinetic equation when truncated at the 0 -th cyclotron harmonic. The 0 -th harmonic of $\mathrm{CKinCH}$ represents the low-frequency drift motion while other harmonics represent the high-frequency ion cyclotron motion. The correspondingly reduced gyrokinetic equation (also with parallel motion and variation removed) is given by

$$
D \delta \hat{F}_{k} / D \hat{t}-i \hat{\omega}_{k}^{d} \delta \hat{G}_{k}=-i \hat{\omega}_{*}^{n T} J_{0}(k \rho) \delta \hat{\phi}_{k} n_{0} F_{M}(\hat{\mu})+\sum_{k 1} \hat{b} \cdot\left(\overrightarrow{\hat{k}}_{1} \times \overrightarrow{\hat{k}}_{2}\right) J_{0}\left(k_{1} \rho\right) \delta \hat{\phi}_{k 1} \delta \hat{F}_{k 2}
$$

where $\delta \hat{F}_{k}(\hat{\mu})=\delta \hat{G}_{k}(\hat{\mu})-\left(T_{e} / T_{i}\right) \delta \hat{\phi}_{k} n_{0} F_{M}(\hat{\mu}) J_{0}(k \rho)$, and $J_{0}(k \rho)$ is the Bessel function.

The Poisson equation of gyrokinetics is 
where $2 \pi \int d \hat{\mu} F_{M}(\hat{\mu})\left[1-J_{0}^{2}(k \rho)\right]$ represents the ion polarization. Gyrokinetics nonlinearly conserves the incremental entropy defined as $E=2 \pi \sum_{k} \int_{0}^{\infty} d \hat{\mu}\left(\delta \hat{F}_{k}^{*} \delta \hat{F}_{k}\right)$.

The gyrokinetic particle and energy fluxes are

$$
\left[\hat{\Gamma}, \hat{Q}^{\perp}\right]=\operatorname{Re} 2 \pi \int_{0}^{\infty} d \hat{\mu} \sum_{k}\left[1,\left(T_{i} / T_{e}\right) \hat{\mu}\right] i \hat{k}_{y} \delta \hat{\phi}_{k}^{*}\left[J_{0}(k \rho) \delta \hat{G}_{k}(\hat{\mu}) / n_{0}\right]
$$

\subsection{Electron motion equations}

In this paper the electron response is given by the collisional drift wave (CDW) electron model which is essentially the Hasegawa-Wakatani ${ }^{10}$ electron model with a grad- $B$ drift added.

$$
D \delta \hat{n}_{k}^{e} / D \hat{t}+i \hat{\omega}_{d}^{e} \delta \hat{n}_{k}^{e}+\alpha_{A}\left(\delta \hat{n}_{k}^{e}-\delta \hat{\phi}_{k}\right)=-i\left(\hat{\omega}_{*}^{n}-\hat{\omega}_{d}^{e}\right) \delta \hat{\phi}_{k}+\sum_{k 1} \hat{b} \cdot\left(\overrightarrow{\hat{k}} \times \overrightarrow{\hat{k}}_{2}\right) \delta \hat{\phi}_{k 1} \delta \hat{n}_{k 2}^{e}
$$

where $\hat{\omega}_{*}^{n} \Rightarrow \hat{k}_{y}\left(a / L_{n}\right)$ is the density gradient drive frequency. $\hat{\omega}_{d}^{e} \Rightarrow 2 \hat{k}_{y}(a / R)$ is the grad- $B$ drift frequency. The factor 2 here is again used to compensate for the parallel motion curvature drive. $\alpha_{A}=\hat{k}_{\|}^{2} /\left[\hat{v}_{e i}\left(m_{e} / m_{i}\right)\right]$ is the CDW adiabaticity parameter used for controlling the strength of the turbulence. $\hat{k}_{\|}=a / R q$ is the parallel wave number and $\hat{v}_{e i}$ is the electron-ion collision frequency. $\alpha_{A}$ is derived from Ohm's law in the parallel direction. Large $\alpha_{A}$ leads to lower turbulence level adiabatic electron response. Small $\alpha_{A}$ corresponds to high turbulence level contributed by the resistivity interchange modes, which are strongly driven at long wave length (small $\hat{k}_{y}$ ) regime. While the overall sign of the nonlinear terms has no effect on the time average transport, it is very important that the electron $E \times B$ nonlinearity have the correct relative sign as the ion $E \times B$ nonlinearity. 


\section{Numerical methods}

\section{1. $\mu$ discretization}

The integration along $\mu$-direction is numerically expressed as:

$$
2 \pi \int_{0}^{\infty} d \hat{\mu} \delta \hat{F}_{k}^{n}(\hat{\mu})=\sum_{j=1}^{j=N_{\mu}} \delta \hat{F}_{k}^{n}\left(\hat{\mu}_{j}\right) W_{j}
$$

We split the interval of integration in $\hat{\mu}$ into two regions: $\left[0, \hat{\mu}_{\max }\right)$ and $\left[\hat{\mu}_{\max }, \infty\right)$. The first region is described by $\hat{\mu}_{j}\left(j=1,2, \ldots, N_{\mu}-1\right)$, and each of the $\mu$-grid points represents the center of a "bin" with "bin weight" $W_{j}$. The corresponding modified bin weights $\bar{W}_{j}=W_{j} F_{M}\left(\hat{\mu}_{j}\right)$ satisfy $\bar{W}_{1}=\bar{W}_{2}=\ldots=\bar{W}_{N_{\mu}-1}$, which means the weights $W_{j}$ are chosen such that for a Maxwellian distribution each $\mu$-grid bin is distributed with the same percentage of the distribution. The second region is represented by the last grid point $\hat{\mu}_{N_{\mu}}$, and we set $\hat{\mu}_{N_{\mu}}=\hat{\mu}_{\max }$. The weight of last grid point evaluates the infinite integral according to $2 \pi \int_{\hat{\mu}_{N_{\mu}}}^{\infty} d \hat{\mu} F_{M}(\hat{\mu})=\bar{W}_{N_{\mu}}$. Setting the modified bin weight of last grid point equal to those of all other grid points $\bar{W}_{N_{\mu}}=\bar{W}_{j} \quad\left(j=1,2, \ldots, N_{\mu}-1\right)$ is a reasonable and satisfactory choice to get good $\mu$-grid convergence (see Sec. 4.2). Thus the weights of all the grid points have the desirable property that $2 \pi \int_{0}^{\infty} d \hat{\mu} F_{M}(\hat{\mu})=\sum_{j=1}^{j=N_{\mu}} W_{j} F_{M}\left(\hat{\mu}_{j}\right)$ $=\sum_{j=1}^{j=N_{\mu}} \bar{W}_{j}=1$. An eight $\mu$-grid example is listed in Table 2. This $\mu$-discretization method is essentially equivalent to the Maxwellian energy space discretization method introduced in Ref. [11]. 
Table 2

Sample magnetic moment $\mu$-grids and weights

\begin{tabular}{lcl}
\hline$j$ & $\hat{\mu}_{j}$ & $\bar{W}_{j}$ \\
\hline 1 & 0.066765696 & 0.1249072 \\
2 & 0.2106067 & 0.1248763 \\
3 & 0.3788429 & 0.1248270 \\
4 & 0.5815754 & 0.1247410 \\
5 & 0.8369882 & 0.1245700 \\
7 & 1.183562 & 0.1241478 \\
8 & 1.732868 & 0.1225323 \\
\hline
\end{tabular}

\subsection{The $\mu$-derivative operator}

The $\mu$-derivative operator $D_{j j^{\prime}}^{\mu}$ is defined as

$$
\left.\partial_{\hat{\mu}} V(\hat{\mu})\right|_{j}=\sum_{j^{\prime}=1}^{j^{\prime}=N_{\mu}} D_{j j^{\prime}}^{\mu} V\left(\hat{\mu}_{j^{\prime}}\right)
$$

where $V(\hat{\mu})$ is an arbitrary function of $\hat{\mu} . D_{j j^{\prime}}^{\mu}$ is required to preserve the integration by parts so as to conserve the incremental entropy. For arbitrary functions $U(\hat{\mu})$ and $V(\hat{\mu})$, the discretized integration by parts is expressed as:

$\sum_{j} W_{j} U\left(\hat{\mu}_{j}\right) \sum_{j^{\prime}} D_{j j^{\prime}}^{\mu} V\left(\hat{\mu}_{j^{\prime}}\right)=\left.U(\hat{\mu}) V(\hat{\mu})\right|_{\hat{\mu}_{1}} ^{\hat{\mu}_{N^{\mu}}}-\sum_{j} W_{j} V\left(\hat{\mu}_{j}\right) \sum_{j^{\prime}} D_{j j^{\prime}}^{\mu} U\left(\hat{\mu}_{j^{\prime}}\right)$. Since numerically $\hat{\mu}_{1}$ and $\hat{\mu}_{N_{\mu}}$ cannot get to 0 and $\infty$, the boundary term $\left.U(\hat{\mu}) V(\hat{\mu})\right|_{\mu_{1}} ^{\hat{\mu}_{N_{\mu}}}$ appears to be non-zero. In order to get rid of the non-zero boundary term, the $\mu$-derivative operator $D_{j j^{\prime}}^{\mu}$ must satisfy the remaining part of the above equation: $\sum_{j} W_{j} U\left(\hat{\mu}_{j}\right) \sum_{j^{\prime}} D_{j j^{\prime}}^{\mu} V\left(\hat{\mu}_{j^{\prime}}\right)=-\sum_{j} W_{j} V\left(\hat{\mu}_{j}\right) \sum_{j^{\prime}} D_{j j^{\prime}}^{\mu} U\left(\hat{\mu}_{j^{\prime}}\right)$ which then can be reduced to:

$$
W_{j} D_{j j^{\prime}}^{\mu}=-W_{j^{\prime}} D_{j^{\prime} j}^{\mu}
$$




\subsection{Linear eigenvalue solver}

$\mathrm{CKinFH}$ is taken as an example to illustrate the rCYCLO eigenvalue solver. The Poisson 
equation Eq. (9) indicates that the electric field $\delta \hat{\phi}_{k}$ is functions of $\delta \hat{F}_{k}^{0}(\hat{\mu})$. Then we can obtain the dispersion matrix $M_{j j^{\prime}}^{n n^{\prime}}$ and the linear eigenvalue equation Eq. (21) through combining the linear parts of Eq. (4) and Eq. (15) with Eq. (9).

$$
-i \hat{\omega} \delta \hat{F}_{k}^{n}\left(\hat{\mu}_{j}\right)=\sum_{n^{\prime}=-N_{\alpha}+1}^{n^{\prime}=N_{\alpha}-1} \sum_{j^{\prime}=0}^{j^{\prime}=N_{\mu}} M_{j j^{\prime}}^{n n^{\prime}}(\hat{k}) \delta \hat{F}_{k}^{n^{\prime}}\left(\hat{\mu}_{j^{\prime}}\right)
$$

where $j=1,2,3 \ldots N_{\mu}$ represent the ion, and $j=0$ represents the electron (for example: $\left.\delta \hat{F}_{k}^{0}\left(\hat{\mu}_{0}\right)=\delta \hat{n}_{k}^{e}\right)$. The linear frequency and growth rate are just the eigenvalues (solved by the numerical library LAPACK) of the dispersion matrix $M_{j j^{\prime}}^{n n^{\prime}}$. It is the same way for gyrokinetics and $\mathrm{CKinCH}$ to solve their linear frequency and growth rate as $\mathrm{CKinFH}$.

\subsection{Time advance method}

Again taking CKinFH as an example, the time evolution equation corresponding to Eq. (21) is:

$$
D \delta \hat{F}_{k, j}^{n} / D \hat{t}=\sum_{n^{\prime}=-N_{\alpha}+1}^{n^{\prime}=N_{\alpha}-1} \sum_{j^{\prime}=0}^{j^{\prime}=N_{\mu}} M_{j j^{\prime}}^{n n^{\prime}}(\hat{k}) \delta \hat{F}_{k, j^{\prime}}^{n^{\prime}}+{ }^{N L} S_{k, j}^{n}
$$

The $\Omega^{*}$ terms result in the large entries in $M_{j j^{\prime}}^{n n '}$ with a wide range of eigenvalues making it a stiff matrix. The stiff matrix requires an implicit time advance. Thus rCYCLO has a time centered implicit linear advance and an explicit nonlinear advance. The perturbed distribution function of next time step $\delta \overline{\hat{F}}_{k, j}^{n}$ can be obtained by multiplying the invers matrix $\left[R^{-1}\right]_{j j^{\prime}}^{n n^{\prime}}$ on $\bar{S}_{k, j}^{n}$.

$$
\delta \overline{\hat{F}}_{k, j}^{n}=\sum_{n^{\prime}=-N_{\alpha}+1}^{n^{\prime}=N_{\alpha}-1} \sum_{j^{\prime}=0}^{j^{\prime}=N_{\mu}}\left[R^{-1}\right]_{j j^{\prime}}^{n n^{\prime}}(\hat{k}) \bar{S}_{k, j^{\prime}}^{n^{\prime}}
$$

where $\bar{S}_{k, j}^{n}$ and $R_{j j^{\prime}}^{n n^{\prime}}$ are given by

$$
\begin{aligned}
& \bar{S}_{k, j}^{n}=\delta \hat{F}_{k, j}^{n}+(d \hat{t} / 2) \sum_{n^{\prime}=-N_{\alpha}+1}^{n^{\prime}=N_{\alpha}-1} \sum_{j^{\prime}=0}^{j^{\prime}=N_{\mu}} M_{j j^{\prime}}^{n n^{\prime}}(\hat{k}) \delta \hat{F}_{k, j^{\prime}}^{n^{\prime}}+(d \hat{t}){ }^{N L} S_{k, j}^{n} \\
& R_{j j^{\prime}}^{n n^{\prime}}(\hat{k})=\delta_{n^{\prime}}^{n} \boldsymbol{\delta}_{j^{\prime}}^{j}-(d \hat{t} / 2) M_{j j^{\prime}}^{n n^{\prime}}(\hat{k})
\end{aligned}
$$


Here $\delta \hat{F}_{k, j}^{n}$ is the perturbed distribution function at the previous time step. ${ }^{N L} S_{k, j}^{n}$ is the value of the nonlinear terms on the previous time step. The Poisson equation Eq. (9) is used to update $\delta \hat{\phi}_{k}$ at the end of each time step. The linear calculation of Poisson equation requires collective communication over the $\mu, n$ dimensions at each $k x$ and $k y$ grid. Therefore, the linear calculation of Eq. (23a) and (23b) is parallelized in $k_{x}$ and $k_{y}$ dimensions. Each of linear $k$ mode is assigned its own processor.

\section{Simulation results}

\subsection{Linear and nonlinear simulations of the standard case}

The parameters of the standard case are listed in Table 3. All calculation in this paper is performed using these parameters unless specified otherwise.

Table 3

The physics parameters of the standard case

\begin{tabular}{lllllllll}
\hline$a / L_{n}$ & $a / L_{T i}$ & $\alpha_{A}$ & $a / R$ & $\mu_{L K}$ & $\mu_{H K}$ & $\Omega^{*}$ & $\hat{\lambda}_{D}$ & $C_{D W}$ \\
\hline 4.00 & 4.00 & 1.0 & 0.3 & 0.05 & 0.3 & 10 & 0.1 & 1 \\
\hline
\end{tabular}

The grid parameters of the standard case

\begin{tabular}{cccc}
\hline$N_{k}$ & $\hat{k}_{x}^{\mathrm{max}}\left(=\hat{k}_{y}^{\mathrm{max}}\right)$ & $N_{\mu}$ & $N_{\alpha}$ \\
\hline $21 \times 21$ & 1.5 & 21 & 5
\end{tabular}


Grid number for a standard case is $N_{k x} \times N_{k y} \times\left(2 N_{\alpha^{-}}-1\right) \times\left(N_{\mu}+1\right)=21 \times 21 \times 9 \times 22=87318$. The $\mu$-grid number 22 equals to $N_{\mu}=21$ for ion plus 1 for electron. The possible maximum processor number for the standard case is $\left(2 N_{\alpha}-1\right) \times\left(N_{\mu}+1\right)=9 \times 22=198$ in the rCYCLO parallel process program. Figure. 1 shows the linear growth rate $\hat{\gamma}$ of the leading low-frequency mode and the corresponding frequency $\hat{\omega}$ using the eigenvalue solver. $\hat{\gamma}$ and $\hat{\omega}$ of gyrokinetics are constant with respect to $\Omega^{*}$. (a) and (b) represent gyrokinetics (GK), (c) and (d) represent CKinFH with $N_{\alpha}=5$, (e) and (f) represent CKinFH with $N_{\alpha}=8$. At least $N_{\alpha}=5$ is required to get the proper gyro-cut-off beyond $\rho_{i} \cdot k_{\perp} \sim O(1)$. The $\hat{\gamma}$ of GK in (a) and of CKinFH in (c) are close to identical. However $\hat{\omega}$ of the $N_{\alpha}=5 \mathrm{CKinFH}$ high $-k$ part $\left(\left|\hat{k}_{x}\right|>1.0\right.$ or $\left.\left|\hat{k}_{y}\right|>1.0\right)$ in (d) is different from $\hat{\omega}$ of GK in (b). This difference will disappear when more harmonics are retained such as $\hat{\omega}$ of $N_{\alpha}=8$ in (f). Since the different high- $k$ part is strongly damped by high- $k$ damping $\mu_{H K} \hat{k}^{4}$ and appears stable, therefore the $N_{\alpha}=5$ is chosen as the standard case is good enough. The undamped leading low-frequency drift-waves are unstable while the undamped high-frequency ion cyclotron modes are marginally stable. The addition of the low- $k$ damping $\mu_{L K} / \hat{k}^{2}$ (high- $k$ damping $\mu_{H K} \hat{k}^{4}$ ) stabilizes the low- $k$ (high- $k$ ) modes providing a sink of incremental entropy. The nonlinear terms transfer the incremental entropy from the unstable source modes to the stable sink modes in $k$ space. Without the artificial damping the high-frequency ion cyclotron modes are neutrally stable, while the low-frequency ITG drift modes (in particular) come in reactively unstable and stable pairs. 

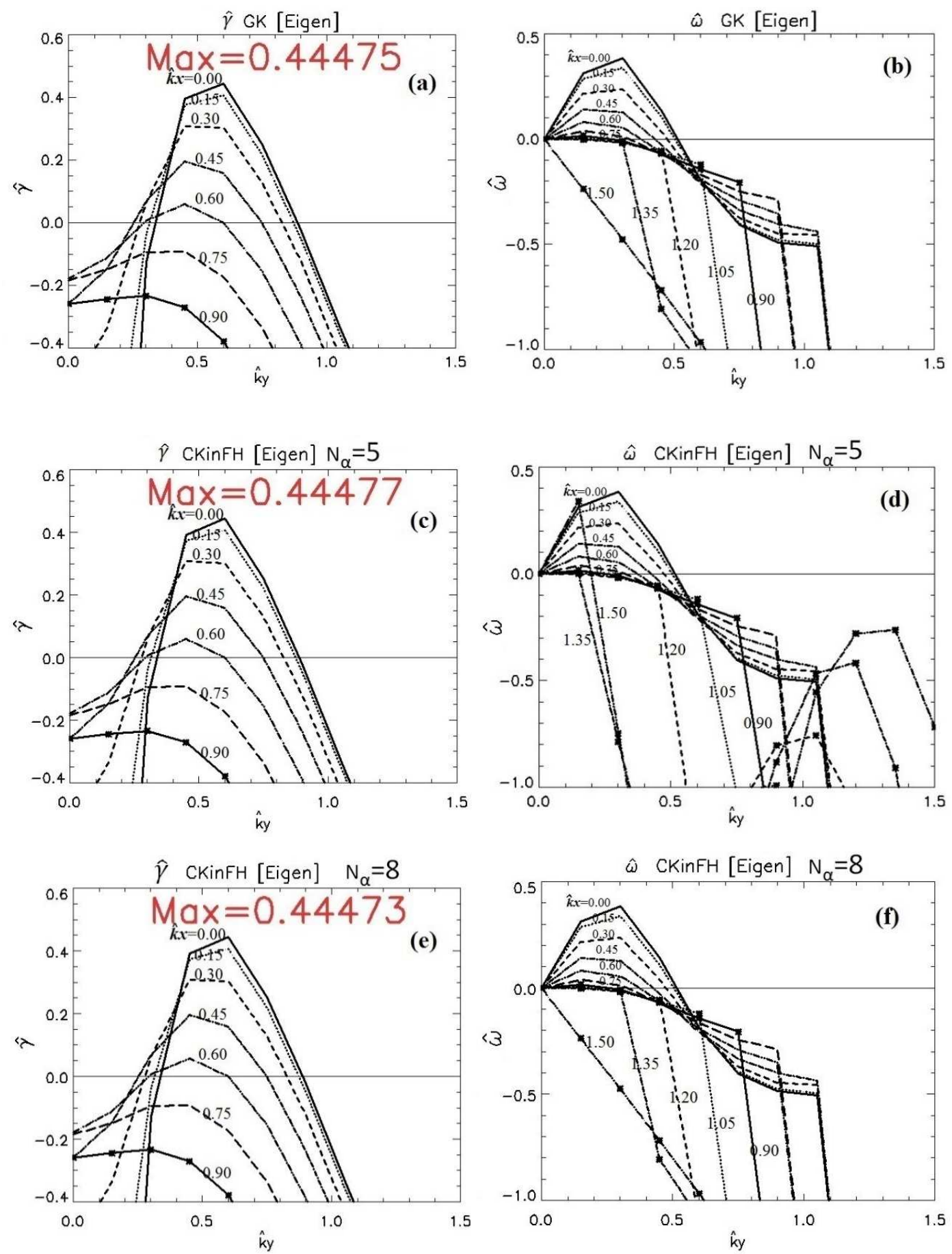

Fig. 1. The most unstable linear growth rate $\hat{\gamma}$ and frequency $\widehat{\omega}$ spectrums solved by the eigenvalue solver. (a) and (b) represent GK; (c) and (d) represent CKinFH with $N_{\alpha}=5$; (e) and (f) represent CKinFH with $N_{\alpha}=8$. The red numbers record the growth rate of the most unstable mode. Only the $k_{x}>0$ and $k_{y}>0$ modes are presented. 
Fig. 2 illustrates the nonlinear saturation processes in the time initial evolution of the standard case. The time average of the energy diffusivity over the saturated time is $\hat{\chi}^{G K}=13.1 \pm 0.1$ for gyrokinetics, and $\hat{\chi}^{C K}=4.05 \pm 0.09$ for cyclokinetics respectively. The corresponding particle diffusivity is $\hat{D}^{G K}=9.8 \pm 0.4$ for gyrokinetics, and $\hat{D}^{C K}=3.5 \pm 0.1$ for cyclokinetics respectively. The time step of the standard case is $\Delta \hat{t}=0.001 \mathrm{a} / \mathrm{c}_{s}$ for gyrokinetics, and $\Delta \hat{t}$ $=0.0001 \mathrm{a} / \mathrm{c}_{s}$ for cyclokinetics respectively. The time step is chosen to conserve the incremental entropy with the error less than a percent when no energy input and damping are added. In general the time step should be smaller when $\Omega^{*}$ is higher e.g. $\Delta \hat{t}=0.00001 a / c_{s}$ is chosen for the cyclokinetic case at $\Omega^{*}=100$. We have verified that the cyclokinetic transport level is insensitive to the artificial damping rates of IC modes as long as IC modes are less unstable than low-frequency drift modes. Fig. 3 presents the frequency spectrums of $\hat{\chi}^{C K}$ and $|\hat{\phi}|^{2}$ of $\mathrm{CKinCH}$ standard case during the saturated time period, which clearly shows the low-frequency drift-wave modes and the six harmonics of high-frequency ion cyclotron modes with $\hat{\omega}$ nonlinearly shifted from the linear $\pm 10, \pm 20$ and \pm 30 values. The linearly damped high-frequency modes are nonlinearly driven by the low-frequency unstable drift modes. The low-frequency unstable drift modes account for most (e.g. 86\% within $|\hat{\omega}| \leq 2)$ of the transport.
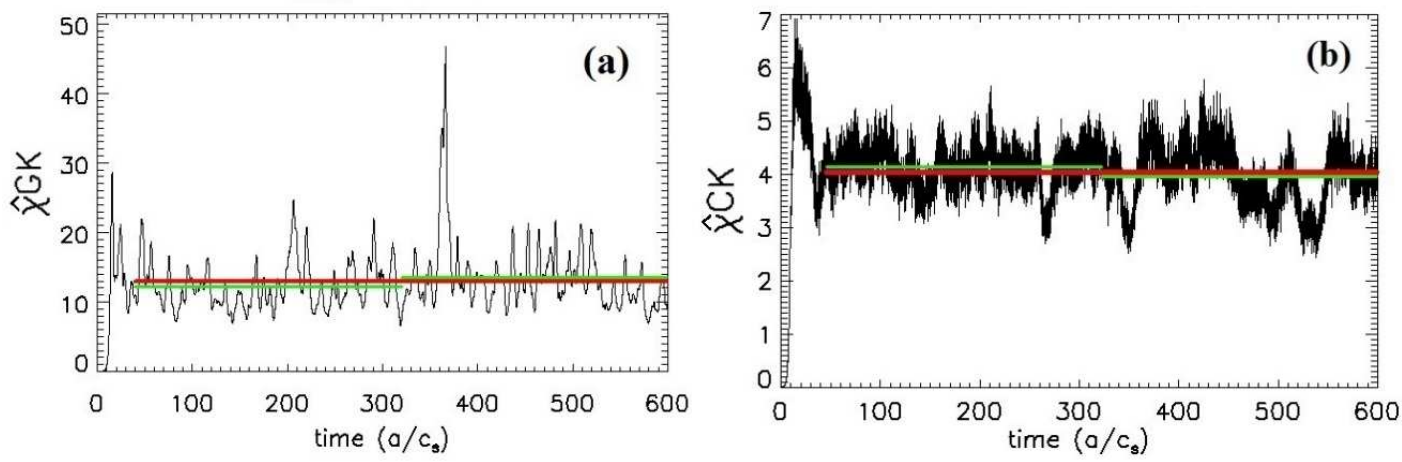

Fig. 2. The time evolution of turbulent energy diffusivity of the GK and CKinFH standard case. The average energy diffusivity over the saturated period of time is (a) $\hat{\chi}=13.1 \pm 0.1$ for GK, (b) $\hat{\chi}=4.1 \pm 0.1$ for $\mathrm{CKinFH}$. The red and green lines represent the average over the all saturated time and the half period of saturated time respectively. 

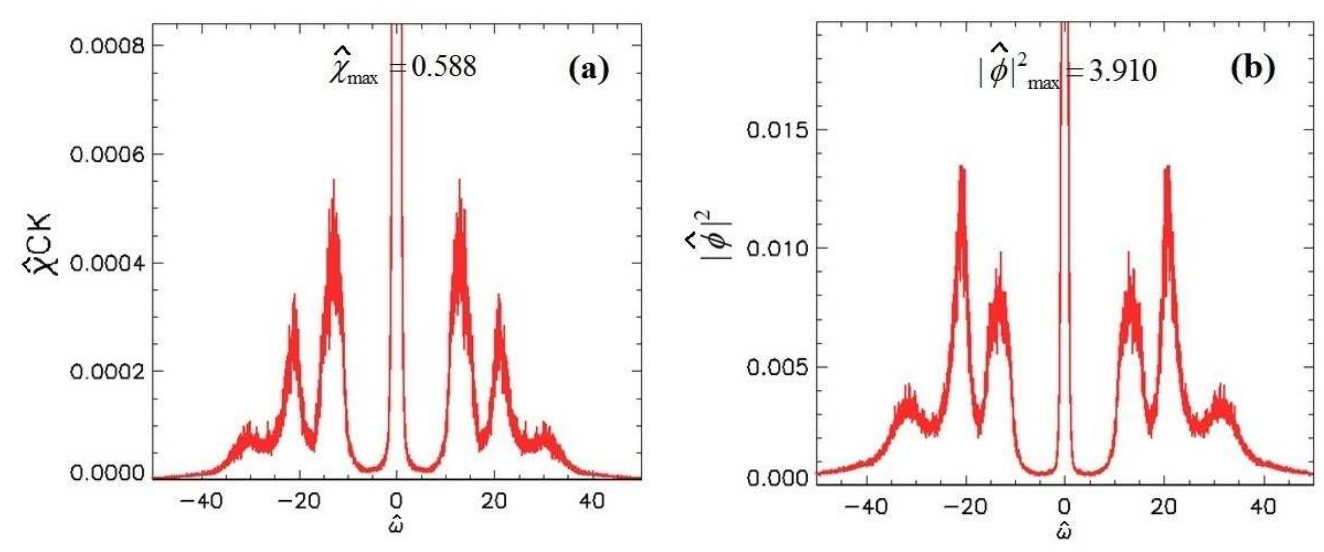

Fig. 3. The frequency spectrum for the CKinFH standard case (summed over all k) during the saturated time. (a) $\hat{\chi}^{C K}$ versus $\widehat{\omega}$, and (b) $|\hat{\phi}|^{2}$ versus $\widehat{\omega}$. The drift-wave modes ( $\widehat{\omega}$ close to 0 ) and the six harmonics of the IC modes ( $\widehat{\omega}$ around $\pm 10, \pm 20$ and \pm 30$)$ are clearly shown.

The two kinds of low-frequency drift modes, ITG modes and CDW modes, are clearly described in Fig. 4. We use the standard GK case (here the same results as CKinFH case), and fix $\hat{k}_{x}=0$ and $\hat{k}_{y}=0.45$ with $\mu_{L K}=0$ and $\mu_{H K}=0$ in Fig. 4 . The negative frequency ITG modes are slightly unstable and almost not influenced by the change of $\alpha_{A}$ [the $\hat{\gamma}$ of ITG modes has been multiplied by 10 in Fig. 4(a)]. The positive frequency CDW modes are dominant. The low-collisional (large $\alpha_{A}$ ) electron modes approach to adiabatic limit, while the highly collisional (small $\alpha_{A}$ ) electron modes will switch to the more unstable resistive-g modes. Since the growth rates of the CDW modes decrease much faster than the ITG modes when $\hat{k}_{x}$ and $\hat{k}_{y}$ increase, the dominant modes will switch from CDW modes to ITG modes when either $\hat{k}_{x}$ or $\hat{k}_{y}$ increases larger than 1.0. The jumps in the frequency spectrum of Fig. 1 (b), (d) and (f) illustrate this mode switch phenomenon. 


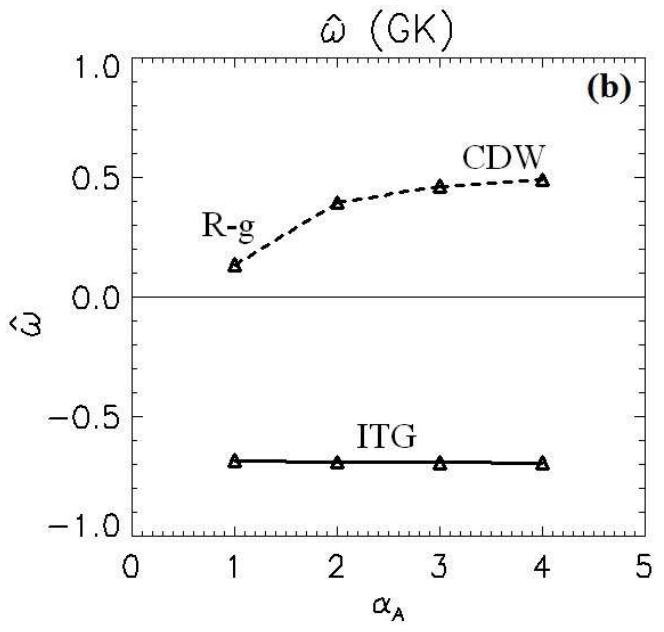

Fig. 4. (a) The linear growth rate $\hat{\gamma}$ and (b) frequency $\widehat{\omega}$ of the standard GK case versus $\alpha_{A}$ at the fixed point $\hat{k}_{x}=0$ and $\hat{k}_{y}=0.45$ with $\mu_{L K}=0$ and $\mu_{H K}=0$. The $\widehat{\omega}$ of ITG modes is negative and the $\hat{\gamma}$ is small [the $\hat{\gamma}$ of ITG modes has been multiplied by 10 in (a)]. The $\widehat{\omega}$ of CDW modes is positive. CDW modes will switch to more unstable resistive-g modes with the smaller $\alpha_{A}$.

\subsection{Mode and grid number convergence test}

Grid convergences in $k$-space, $\mu$-space, and Fourier harmonics are demonstrated here. During the grid convergence test, only one grid parameter is changed while all other grid parameters keep the same as standard case. Fig. 5(a) displays the GK energy diffusivity $\hat{\chi}$ versus number of $k$-modes, which demonstrates good $k$-space grid convergence. The number of modes $N_{k}$ is increased from $11 \times 11=121$ to $29 \times 29=841$ with fixing $\hat{k}_{x}^{\max }=\hat{k}_{y}^{\operatorname{mx}}=1.5$. Grids converge after $N_{k}=17 \times 17=289$, which indicates that $N_{k}=21 \times 21=441$ is good enough for the standard case. Fig. 5 (b) shows the GK energy diffusivity $\hat{\chi}$ versus $\mu$-grid number $N_{\mu}$, which converges after $N_{\mu}=11$ with $N_{\mu}=21$ chosen as the standard case parameter. Here and below the standard case is always marked by red circle. 


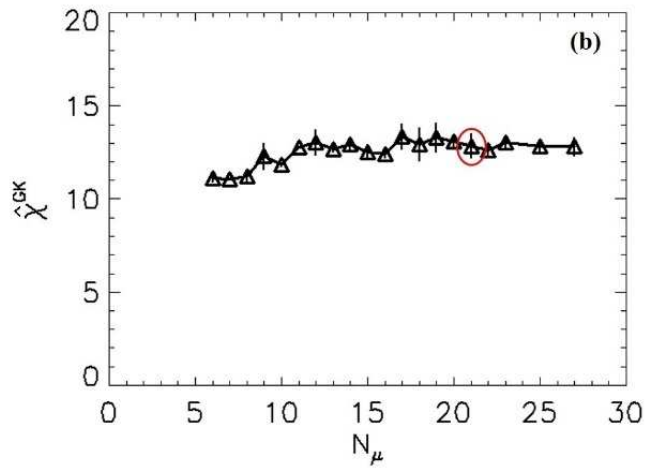

Fig. 5. (a) The gyrokinetic energy diffusivity $\hat{\chi}$ versus the number of $k$-modes $N_{k}$ for the mode number convergence test. The standard case is chosen as $N_{k}=21 \times 21=441$. (b) The gyrokinetic energy diffusivity $\hat{\chi}$ versus $N_{\mu}$ for the $\mu$-grid convergence test. The standard case is chosen as $N_{\mu}=21$.

Fig. 6 shows the CKinFH energy diffusivity $\hat{\chi}$ versus number of harmonics of gyro motion (calculated by $2 N_{\alpha^{-}}-1$ ), which converges after 7 with 9 chosen for the standard case. Fig. 7 illustrates the CKinFH energy diffusivity $\hat{\chi}$ versus $N_{\mu}$. When $N_{\mu}$ increases, the resolution of $\mu$ grid increases fast while the $\mu_{\max }$ increases slow. Note that the odd and even $\mu$-gird numbers follow different convergence processes at $\Omega^{*}=10$ (the standard case parameter) which is shown in Fig. 7(a). The odd $N_{\mu}$ converges faster than the even, and finally they approach to a close level, therefore we choose the odd number $N_{\mu}=21$ as the standard case. We do not understand why the odd grid converges faster than the even grid. We choose the odd number as the standard case for its faster convergence. Using the even grid would not materially change any physically meaningful conclusion. The separated convergence between the odd and even $N_{\mu}$ is caused by the inaccuracy of the $\mu$-derivative operation as discussed in Section 3.2. This separated convergence effect will be suppressed when either the $\mu$-grids are more closely spaced by increasing $N_{\mu}$, or the coupling between the low-frequency motion and the high-frequency motion is decreased by increasing the relative ion cyclotron frequency $\Omega^{*}$. As evidence, Fig. $7\left(\right.$ b) shows that when a higher $\Omega^{*}=30$ is applied, the odd and even convergence processes almost recover each other. This result also indicates that the inaccuracy of the $\mu$-derivative operation is just some error in "scrambling" and not so important. 


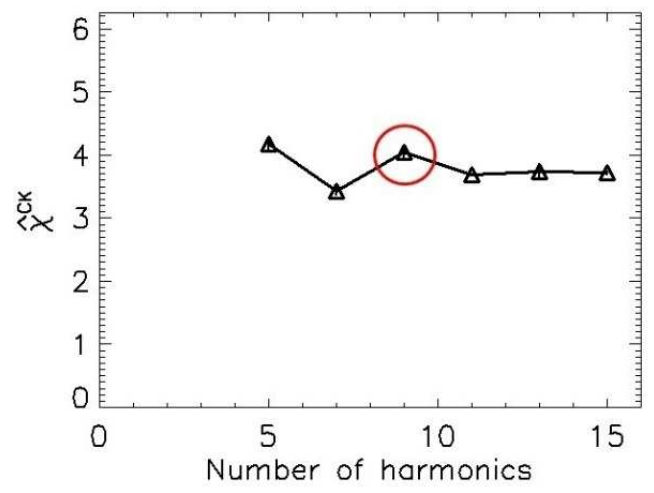

Fig. 6. The cyclokinetic energy diffusivity $\hat{\chi}$ versus the number of harmonics of gyro motion (calculated by $\left.2 N_{\alpha}-1\right)$ for the harmonic number convergence test. The standard case is chosen as 9.
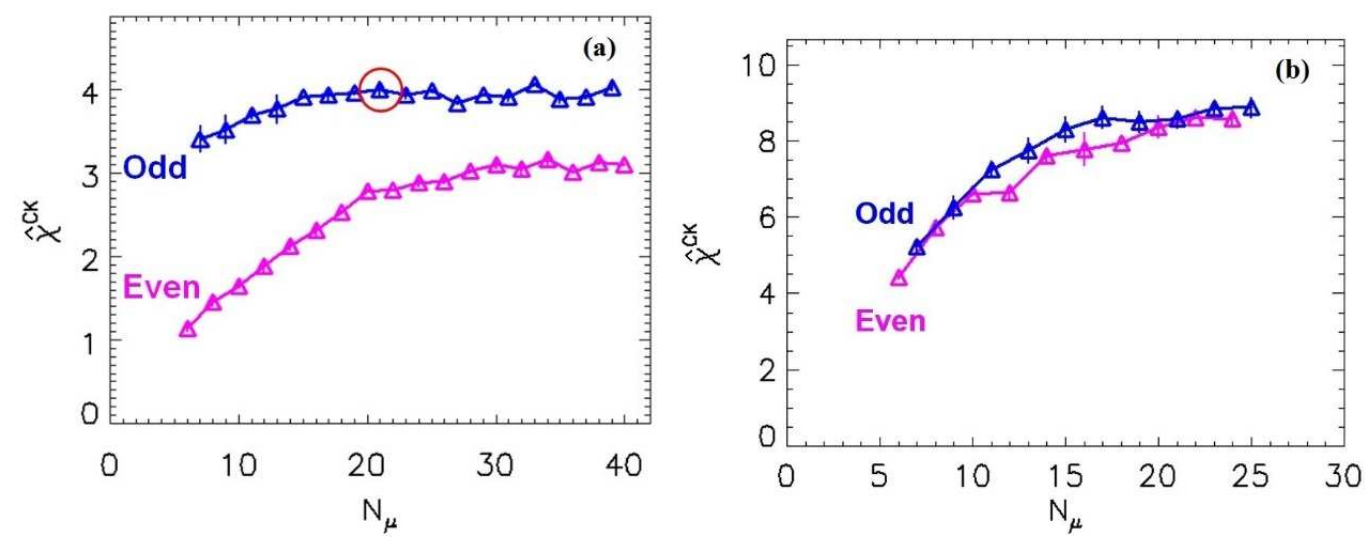

Fig. 7. The cyclokinetic energy diffusivity $\hat{\chi}$ versus $N_{\mu}$, where (a) the odd and even $\mu$ gird numbers follow the different convergence processes at $\Omega^{*}=10$ (the standard case parameter); (b) the odd and even convergence processes almost recover each other at $\Omega^{*}=30$.

\subsection{Code verification}

In testing the high turbulence level breakdown of low-frequency gyrokinetics against high-frequency cyclokinetics, rCYCLO must verify: (1) the low-frequency cyclokinetic linear rates are not significantly different from the gyrokinetic rates (even at $\Omega^{*}$ values as low as 10 ); and (2) at sufficiently large $\Omega^{*}$ and sufficiently low turbulence level the nonlinear gyrokinetic simulations must recover the cyclokinetic transport levels as required.

The linear portion of the initial value time advance in Section 3.4 has been tested for accuracy 
against the eigenvalue solver in Section 3.3. The growth rates from the initial value method recover the results from the eigenvalue solver, and the low-frequency mode growth rates of gyrokinetics recover both $\mathrm{CKinCH}$ and $\mathrm{CKinFH}$. These linear verifications ensure a reliable time evolution.

As further verification, the rCYCLO code can also treat drift-kinetics in comparison to gyrokinetics. The drift-kinetic equation, which is valid only for very low- $k$ and low-frequency motion, can be obtained by adding a small parameter $G$ into the Bessel functions of the gyrokinetic equations: $J_{0}(G k \rho) \rightarrow 1$ and $\left[1-J_{0}^{2}(G k \rho)\right] / G^{2} \rightarrow(k \rho)^{2} / 4$ as $G \rightarrow 0$. Fig. 8(a) illustrates that drift kinetics produces higher turbulence transport than gyrokinetics, which is first noted in the GYRO $^{1}$ simulations of Ref [3]. The physical reason is that drift-kinetics has no gyro-cut-off on the high- $k$ instability. This is one of our motivations to develop rCYCLO discussed in Section 1. Moreover, the relative difference between drift-kinetics and gyrokinetics is less for high turbulence levels (small $\alpha_{\mathrm{A}}$ ) because, as a general rule, the spectral peak (in turbulence intensity and transport) always shifts to lower $k$ with higher transport levels as shown in Fig. 8(b). The standard case parameters are applied in Fig. 8.
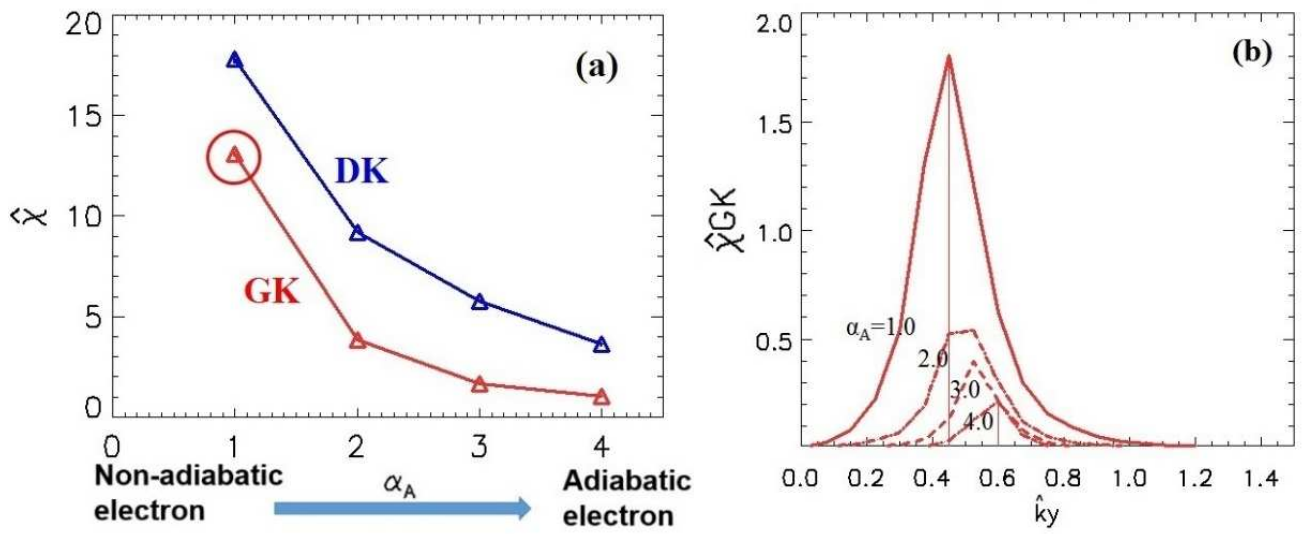

Fig. 8. (a) The energy diffusivity of drift kinetics and gyrokinetics versus $\alpha_{A}$, where drift kinetic diffusivity is higher than gyrokinetics. (b) The GK energy diffusivity spectral peak shifts to low- $k$ when turbulence level increases. 
Fig. 9 illustrates that the nonlinear verification of the gyrokinetic transport level recovers the cyclokinetics at high $\Omega^{*}$ values and low turbulence level as expected. Notice that the cyclokinetic transport level is lower, not higher, than the gyrokinetic transport at low- $\Omega^{*}$ values. The turbulence is drained in frequency space from the unstable low-frequency domain and spreads into the less unstable high-frequency domain. The transport level of cyclokinetics is lower than that of gyrokinetics because the turbulence is drained to the less unstable IC modes.
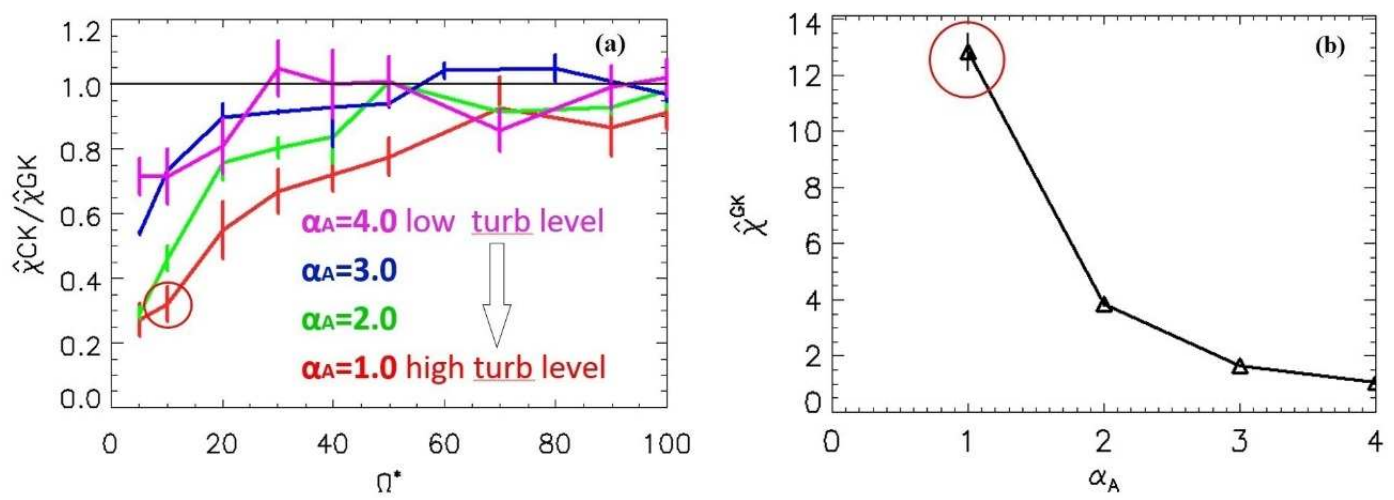

Fig. 9. (a) The energy diffusivity ratio of cyclokinetics over gyrokinetics versus $\Omega^{*}$. The transport level of gyrokinetics recovers cyclokinetics at high- $\Omega^{*}$ values and low turbulence levels. Cyclokinetic transport level is lower than gyrokinetics at low- $\Omega *$ values and high turbulence levels. (b) The gyrokinetic energy diffusivity versus $\alpha_{A}$.

\subsection{Physics discussion}

The most important physical result in this paper is illustrated in Fig. 9(a). This cyclokinetic small $\Omega^{*}=1 / \rho^{*}$ stabilizing effect from coupling to stable high-frequency modes is analogous to the global gyrokinetic large $\rho^{*}$ stabilizing effect from nonlocal turbulence spreading (NTS) ${ }^{12}$. Both L-mode and H-mode DIII-D simulations ${ }^{13}$ with the GYRO code show that the NTS mechanism breaking gyroBohm scaling is due to the nonlocal drainage of turbulence from the unstable regions and spreading to the stable (or less unstable) regions. At larger $\rho^{*}$ where the small "local" eddies become the large "global" eddies, there is a transition from gyroBohm scaling $\chi_{g B}=\left(c_{s} / a\right) \rho_{s}^{2}$ to Bohm scaling $\chi_{B}=\chi_{g B} / \rho^{*}=\chi_{g B} \Omega^{*}$. At smaller $\Omega^{*}$, cyclokinetics transitions in a similar way. 
The turbulence is drained in the frequency space rather than radial space from the unstable low-frequency domain and spreads into the stable high-frequency domain. The NTS paradigm for breaking gyroBohm scaling in the unstable regions $\chi=\hat{\chi}(0) \chi_{g B}\left(1-\rho^{*} / \rho_{c r i t}^{*}\right)$ is represented by Fig. 10(a) taken from Ref. [13]. $\hat{\chi}(0) \chi_{g B}$ represents the local gyro-Bohm limit at vanishing $\rho^{*}$ obtainable from flux-tube simulations. The cyclokinetic high-frequency stabilization can be written analogously $\hat{\chi}^{C K}=\hat{\chi}^{G K}\left(1-\Omega_{c r i t}^{*} / \Omega^{*}\right)$ as represented in Fig. 10(b). The $\Omega_{c r i t}^{*}$ increases when the turbulence level increases as shown in Fig. 9(a). Furthermore, in the stable (or less unstable) region, where the local gyroBohm diffusivity is expected to be smaller, the radially nonlocal NTS mechanism can be represented by $\chi=\hat{\chi}(0) \chi_{g B}\left(1+\rho^{*} / \rho_{\text {crit }}^{*}\right)$ [13]. It has a contribution from super-gyroBohm $\chi_{g B}^{S}$ to gyroBohm $\chi_{g B}$ so that the transport level is higher than the local gyroBohm because the turbulence spreads into this region from a more unstable region. This suggests that if the cyclokinetic high-frequency IC modes are driven unstable, it is reasonable to expect that $\hat{\chi}^{C K}=\hat{\chi}^{G K}\left(1+\Omega_{c r i t}^{*} / \Omega^{*}\right)$ with the cyclokinetic transport level higher than gyrokinetics. Future work will focus on models with high-frequency IC modes driven unstable. 

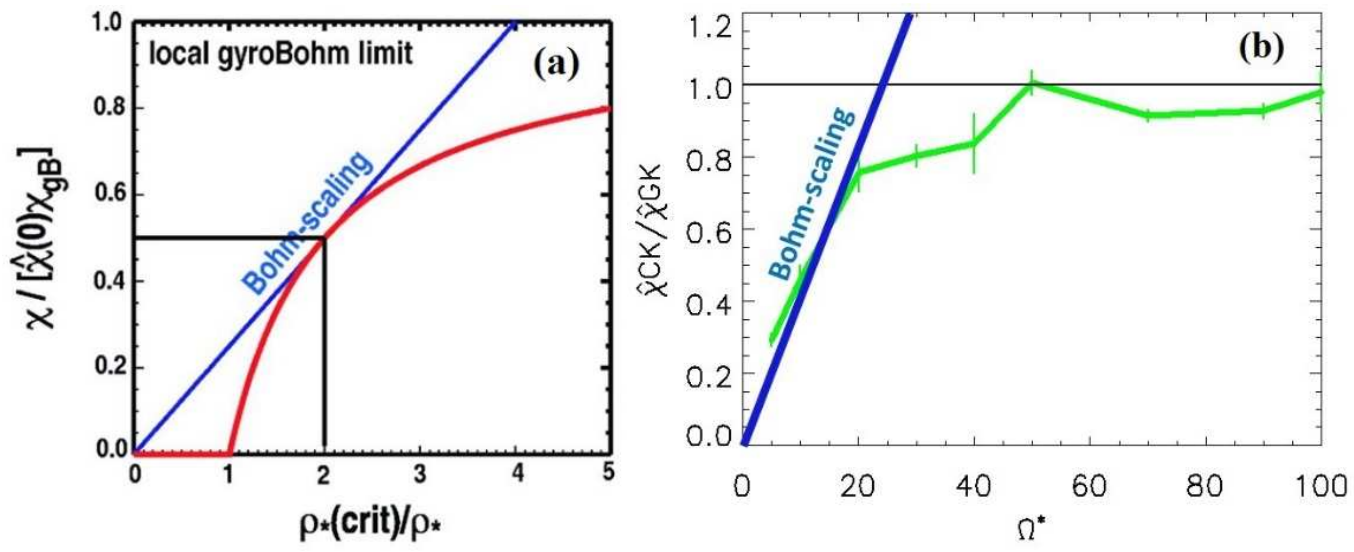

Fig. 10. (a) is from Ref. [13] which shows the rule for breaking gyroBohm scaling by the NTS mechanism. Global gyrokinetic energy diffusivity normalized by gyroBohm level at $\rho^{*} \Rightarrow 0$ vs $\rho_{c r i t}^{*} / \rho^{*}$. (b) Local cyclokinetic energy diffusivity normalized by gyrokinetic diffusivity vs $\Omega^{*}$, which shows the cyclokinetics has the similar rule for breaking gyroBohm scaling by nonlocal turbulence spreading in frequency spectrum.

\section{Conclusions about numerical methods}

In this work, we present the numerical methods for the first nonlinear simulations of cyclokinetic equations. The corresponding code rCYCLO is also introduced, which is a parallel processed, Eulerian code. An implicit time linear advance step with an explicit lag time nonlinear step is applied in rCYCLO. The implicit time linear advance ensures the accuracy of treating the stiff linear dispersion matrix. The explicit time nonlinear advance requires a very tiny time spacing. A novel discretized $\mu$-derivative operator is developed. It is designed to cancel the numerically non-zero boundary terms so as to precisely conserve the incremental entropy. It should be useful to any Eulerian method for solving the ion (or electron) gyro-phase motion in the future. The validation and verification of rCYCLO code and the grid convergence test were also presented. Finally we can add that while the nonlinear simulations of CKinFH is about 10 times faster than those of $\mathrm{CKinCH}$, the linear and nonlinear simulations in $\mathrm{CKinCH}$ reproduce those in $\mathrm{CKinFH}$ to a remarkable accuracy ${ }^{14}$.

\section{Acknowledgements}

We want to thank Dr. Jeff Candy for discussions on the accuracy of spectral derivative operators and Dr. Emily Belli for helpful suggestions reorganizing the writing of this paper. This 
work is supported by China Scholarship Council, NSFC Grant No. 1126114032 and No. 10975012, ITER China Grant No. 2013GB112006 as well as the U.S. Department of Energy Grant No. DE-FG02-95ER54309. 
Ref. [4] gives cyclokinetic equation in cyclotron harmonic form which is listed here for the convenience of readers. The difference between Eq. (A.1) here and Eq. (18c) of Ref. [4] is that the sign of nonlinear terms is flipped, which is caused by the Eq. (11) of this paper corrected a minus sign to the Eq. (12) of Ref. [4]. The non-conservative form of CKinCH is

$$
\begin{aligned}
& D \delta \hat{F}_{k}^{n} / D \hat{t}-i \hat{\omega}_{k}^{d} \delta \hat{G}_{k}^{n}-i n \Omega_{*} \delta \hat{G}_{k}^{n}+J_{n}(k \rho) \delta \hat{\phi}_{k} i \hat{\omega}_{* k}^{n T} n_{0} F_{M}(\hat{\mu}) \\
& =\sum_{n^{\prime}} \sum_{k 1} \hat{b} \cdot \overrightarrow{\hat{k}}_{1} \times \overrightarrow{\hat{k}}_{2}\left[J_{n-n^{\prime}}\left(k_{1} \rho\right)\right] \delta \hat{\phi}_{k 1} \delta \hat{F}_{k 2}^{n^{\prime}} \Delta_{n}^{n^{\prime}}\left(\beta, \beta_{1}, \beta_{2}\right) \\
& +\sum_{n^{\prime}} \sum_{k 1}\left(\hat{k}_{1} / \hat{v}_{\perp}\right)\left\{\left[J_{n-n^{\prime}+1}\left(k_{1} \rho\right)-J_{n-n^{\prime}-1}\left(k_{1} \rho\right)\right] /(2 i)\right\} \delta \hat{\phi}_{k 1}\left(n n^{\prime} \delta \hat{F}_{k 2}^{n^{\prime}}\right) \Delta_{n}^{n^{\prime}}\left(\beta, \beta_{1}, \beta_{2}\right) \\
& +\sum_{n^{\prime}} \sum_{k 1}\left(\hat{k}_{1} / \hat{v}_{\perp}\right)\left\{\left[J_{n-n^{\prime}+1}\left(k_{1} \rho\right)+J_{n-n^{\prime}-1}\left(k_{1} \rho\right)\right] /(2 i)\right\} \delta \hat{\phi}_{k 1}\left(2 \hat{\mu} \partial_{\hat{\mu}} \delta \hat{F}_{k 2}^{n^{\prime}}\right) \Delta_{n}^{n^{\prime}}\left(\beta, \beta_{1}, \beta_{2}\right)
\end{aligned}
$$

The conservative form is

$$
\begin{aligned}
& D \delta \hat{F}_{k}^{n} / D \hat{t}-i \hat{\omega}_{k}^{d} \delta \hat{G}_{k}^{n}-i n \Omega_{*} \delta \hat{G}_{k}^{n}+J_{n}(k \rho) \delta \hat{\phi}_{k} i \hat{\omega}_{* k}^{n T} n_{0} F_{M}(\hat{\mu}) \\
& =\sum_{n^{\prime}} \sum_{k 1} \hat{b} \cdot \overrightarrow{\hat{k}}_{1} \times \overrightarrow{\hat{k}}_{2}\left[J_{n-n^{\prime}}\left(k_{1} \rho\right)\right] \delta \hat{\phi}_{k 1} \delta \hat{F}_{k 2}^{n^{\prime}} \Delta_{n}^{n^{\prime}}\left(\beta, \beta_{1}, \beta_{2}\right) \\
& +\sum_{n^{\prime}} \sum_{k 1}\left(\hat{k}_{1} / \hat{v}_{\perp}\right)\left\{\left[J_{n-n^{\prime}+1}\left(k_{1} \rho\right)-J_{n-n^{\prime}-1}\left(k_{1} \rho\right)\right] /(2 i)\right\} \delta \hat{\phi}_{k 1}\left(n \delta \hat{F}_{k 2}^{n^{\prime}}\right) \Delta_{n}^{n^{\prime}}\left(\beta, \beta_{1}, \beta_{2}\right) \\
& +\sum_{n^{\prime}} \sum_{k 1} \delta \hat{\phi}_{k 1}\left(\mathrm{~T}_{e} / \mathrm{T}_{i}\right) \partial_{\hat{\mu}}\left\{\left[J_{n-n^{\prime}+1}\left(k_{1} \rho\right)+J_{n-n^{\prime}-1}\left(k_{1} \rho\right)\right]\left(\hat{k}_{1} \hat{v}_{\perp}\right) \delta \hat{F}_{k 2}^{n^{\prime}} /(2 i)\right\} \Delta_{n}^{n^{\prime}}\left(\beta, \beta_{1}, \beta_{2}\right)
\end{aligned}
$$

where $\delta \hat{F}_{k}^{n}(\hat{\mu})=\delta \hat{G}_{k}^{n}(\hat{\mu})-J_{n}(k \rho) \delta \hat{\phi}_{k}\left(T_{e} / T_{i}\right) n_{0} F_{M}(\hat{\mu}), \quad$ and $\quad J_{n}\left(k_{1} \rho\right)$ is Bessel function. $\Delta_{n}^{n^{\prime}}\left(\beta, \beta_{1}, \beta_{2}\right) \equiv \exp \left[-i n\left(\beta_{1}-\beta\right)+i^{\prime}\left(\beta_{1}-\beta_{2}\right)\right]$ is a phase coefficient between different wave angles. The perturbed distribution function satisfies the reality condition $\delta \hat{F}_{-k}^{-n}=(-1)^{n}\left[\delta \hat{F}_{k}^{n}\right]^{*}$.

The Poisson equation of $\mathrm{CKinCH}$ is given as

$$
\delta \hat{\phi}_{k}=\frac{\sum_{n} 2 \pi \int d \hat{\mu} J_{n}(k \rho) \delta \hat{F}_{k}^{n}(\hat{\mu}) / n_{0}-\delta \hat{n}_{k}^{e}}{\left(T_{e} / T_{i}\right)\left[1-\sum_{n} 2 \pi \int d \hat{\mu} F_{M}(\hat{\mu}) J_{n}^{2}(k \rho)\right]+\left(\hat{\lambda}_{D}^{2} \hat{k}^{2}\right)} .
$$

The polarization density formally vanishes when all cyclotron harmonics are retained: $\left.\left[1-\sum_{n} 2 \pi \int d \hat{\mu} F_{M}(\hat{\mu}) J_{n}^{2}(k \rho)\right] \rightarrow 0\right|_{n \rightarrow \pm \infty}$.

The ion particle and energy fluxes are 


$$
\left[\hat{\Gamma}, \hat{Q}^{\perp}\right]=\operatorname{Re} 2 \pi \int_{0}^{\infty} d \hat{\mu} \sum_{k} \sum_{n}\left[1,\left(T_{i} / T_{e}\right) \hat{\mu}\right] i \hat{k}_{y} \delta \hat{\phi}_{k}^{*} J_{n}(k \rho) \delta \hat{G}_{k}^{n}(\hat{\mu}) / n_{0} .
$$




\section{References}

${ }^{1}$ J. Candy and R.E. Waltz, Phys. Rev. Lett. 91, 045001 (2003).

${ }^{2}$ C. Holland, A.E. White, et al., Phys. Plasmas 16, 052301 (2009).

${ }^{3}$ R. E. Waltz, "Search for the missing L-mode edge transport and possible breakdown of gyrokinetics", BAPS Series II, Vol. 57, No. 12, (2012) p. 105, DI3-2.

${ }^{4}$ R. E. Waltz and Zhao Deng, Phys. Plasmas 20, 012507 (2013).

${ }^{5}$ R. D. Hazeltine and J. D. Meiss, Plasma Confinement (Addison-Wesley 1992).

${ }^{6}$ W. E. Drummond and M. N. Rosenbluth, Phys. Fluids 5, 1507 (1962).

${ }^{7}$ N. A. Krall and M. N. Rosenbluth, Phys. Fluids 5, 1435 (1962).

${ }^{8}$ N. A. Krall and M. N. Rosenbluth, Phys. Fluids 8, 1488 (1965).

${ }^{9}$ J. Candy and R.E. Waltz, Phys. Plasmas 13, 032310 (2006).

${ }^{10}$ A. Hasegawa and M. Wakatani, Phys. Rev. Lett. 50, 682 (1983).

11 J. Candy and R. Waltz, J. Comput. Phys. 186, 545 (2003).

12 Z. Lin, S. Ethier, T. S. Halm, and W. Tang, Phys. Rev. Lett. 88, 195004 (2002).

${ }^{13}$ R. E. Waltz, J. Candy, and C. C. Petty, Phys. Plasmas 13, 072304 (2006).

${ }^{14}$ Zhao Deng and R. E. Waltz, "Testing the high turbulence level breakdown of low-frequency gyrokinetics against high-frequency cyclokinetic simulations", to be published in Phys. Plasmas. 